\title{
Topical Applications of a Novel Emollient Inhibit Inflammation in Murine Models of Acute Contact Dermatitis
}

\author{
Si Wen $\left(\mathbb{D},{ }^{1}\right.$ Mengke Sun $\left(\mathbb{D},{ }^{2}\right.$ Li Ye $\mathbb{D}^{1},{ }^{1}$ Bin Yang $\mathbb{D}^{1},{ }^{1}$ Lizhi Hu $\mathbb{D},{ }^{2}$ and Mao-Qiang Man $\mathbb{D}^{1}$ \\ ${ }^{1}$ Dermatology Hospital of Southern Medical University, Guangdong 510091, China \\ ${ }^{2}$ Immunology Department, Key Laboratory of Immune Microenvironment and Disease (Ministry of Education), \\ Tianjin Medical University, Tianjin 300070, China
}

Correspondence should be addressed to Lizhi Hu; lizhihu@tmu.edu.cn and Mao-Qiang Man; mqman@hotmail.com

Received 21 January 2021; Revised 6 March 2021; Accepted 7 April 2021; Published 13 April 2021

Academic Editor: Giuseppe Valacchi

Copyright ( $92021 \mathrm{Si}$ Wen et al. This is an open access article distributed under the Creative Commons Attribution License, which permits unrestricted use, distribution, and reproduction in any medium, provided the original work is properly cited.

\begin{abstract}
The benefits of emollients for eczematous dermatitis and psoriasis have been thought to be due to the improvements in epidermal function, including epidermal permeability barrier, stratum corneum hydration, and stratum corneum $\mathrm{pH}$. We determined here whether emollient can direct inhibit cutaneous inflammation. Ear inflammation was induced by topical application of either 12O-tetradecanoylphorbol-13-acetate (TPA) or 1-fluoro-2,4-dinitrofluorobenzene (DNFB). Either 1\% hydrocortisone cream or the novel emollient was applied to the right ear of the mice $45 \mathrm{~min}$ and 2 hours after TPA or DNFB application. The untreated left ear served as untreated controls. Both ear weight and ear thickness were measured 24 hours after TPA and DNFB application. Topical applications of either hydrocortisone cream or emollient significantly decreased both ear thickness and ear weight in comparison to untreated controls. In DNFB model, hydrocortisone significantly lowered expression levels of mRNA for IL- $1 \alpha$, IL- $1 \beta$, and TNF $\alpha$, while the emollient markedly decreased expression levels of IL- $1 \alpha$ and TNF $\alpha$ mRNA. In TPA model, both hydrocortisone and emollient significantly decreased expression levels of IL- $1 \alpha$, IL-1 $\beta$, IL-6, and TNF $\alpha$ mRNA. In parallel, inflammatory infiltration was also reduced by topical applications of either hydrocortisone or emollient. These results demonstrate that this novel emollient can directly inhibit cutaneous inflammation in murine models of both acute irritant contact dermatitis and acute allergic contact dermatitis. However, whether this emollient could also alleviate eczematous dermatitis in humans remains to be explored.
\end{abstract}

\section{Introduction}

Eczematous dermatitis is a common skin disorder, with prevalence of $10 \%$ in adult and as high as $24 \%$ in children aged 67 years $[1,2]$. Although glucocorticoids and immunosuppressants can alleviate eczematous dermatitis, frequent relapse requires long-term treatment, leading an increase in incidence of adverse reactions. However, a number of studies have shown that topical emollients benefit dermatitis although some studies showed no benefits in infants [3-5]. For example, topical emollient alone can improve clinical signs and symptoms of atopic dermatitis [6-8]. The efficacy of emollient is comparable to that of hydrocortisone for atopic dermatitis in humans [9]. Moreover, daily applications of emollient for 6 months can reduce the risk of atopic dermatitis by $50 \%$ in infants [10]. Similarly, Horimukai et al.
[11] showed that topical emollient reduced the incidence of atopic dermatitis by $32 \%$ in infants. Furthermore, topical emollients not only improve atopic dermatitis but also prevent atopic flare by $41 \%$ and delay the flare by 152 days [12]. Additionally, combination of emollient and glucocorticoids can reduce the usage of glucocorticoids, enhance efficacy, and delay relapse in comparison to glucocorticoids alone $[8,13-15]$. Finally, combination therapy of emollient and glucocorticoids increases efficacy for psoriasis, another common inflammatory skin disorder [16-18]. A recent study showed that topical emollient alone delays the relapse of psoriasis [19]. Collectively, this evidence indicates that emollients can alleviate cutaneous inflammation.

Regarding the mechanisms by which emollients improve cutaneous inflammation, it is assumed to be primarily due to the improvements in epidermal permeability barrier function 
and stratum corneum hydration. Indeed, dysfunction in epidermal permeability barrier provokes cutaneous inflammation and predisposes to the development of cutaneous inflammation [20-24]. Likewise, either reduction in stratum corneum hydration or elevation in stratum corneum $\mathrm{pH}$ can also induce or exacerbate cutaneous inflammation [25]. Conversely, acidification of the stratum corneum prevents the development of atopic dermatitis in mice [26, 27]. Thus, emollient-induced improvements in epidermal function can contribute to the alleviation of cutaneous inflammation. However, some emollients contain fatty acids, which can directly inhibit cutaneous inflammation, independent of either epidermal permeability barrier or stratum corneum hydration [28]. In the present study, we determined whether topical applications of a novel emollient containing natural oils can inhibit cutaneous inflammation in mouse models of acute contact dermatitis.

\section{Materials and Methods}

\subsection{Experimental Design}

2.1.1. Animals and Materials. Eight-week old female C57BL/6J were purchased from Guangdong Animal Center (Guangzhou, Guangdong, China) and were fed with mouse diet and water ad libitum. Emollient, YuZe Skin Barrier Recovery Body Lotion ${ }^{\circledR}$, was provided by Jahwa United Company (Shanghai, China). Main ingredients in the emollient include Glycerin, Olea europaea (olive) fruit oil, Carthamus tinctorius (safflower) seed oil, Butyrospermum parkii (shea butter), Persea gratissima (avocado) oil, Oryza sativa (rice) bran oil, sodium stearoyl lactylate, and sodium methyl stearoyl taurate. Hydrocortisone cream (1\%) (Tianjin pharmaceutical, China) was purchased from local pharmacy store. Both 12-O-tetradecanoylphorbol-13-acetate (TPA) and 1-fluoro-2,4-dinitrofluorobenzene (DNFB) were purchased from Merck (Darmstadt, Germany) and Aladdin (Shanghai, China), respectively.

2.1.2. Experimental Protocols. All animal procedures were approved by the Animal Study Subcommittee of the Southern Medical University and performed in accordance with their guidelines. Anti-inflammatory assay was carried out according to the method previously described with slight modification $[29,30]$. Irritant contact dermatitis was induced by a single topical application of $10 \mu \mathrm{l}$ of $0.03 \%$ (wt/vol. in acetone) TPA on the inner and outer surfaces of both ears of mice. Allergic contact dermatitis was induced by single topical application of $20 \mu \mathrm{l}$ of $0.35 \%$ DNFB to the inner and outer surfaces of both ears of mice 5 days following sensitization with $25 \mu \mathrm{l}$ of $0.5 \%$ DNFB. The right ears were treated with either $1 \%$ hydrocortisone or emollient at $45 \mathrm{~min}$ and $2 \mathrm{~h}$ following application of TPA or DNFB while the left ears served as untreated controls. Additional group of normal mice served as normal controls. Twenty-four hours after TPA and DNFB application, ear thickness was measured with a digital caliper (Mitutoyo Corp., Tokyo, Japan), followed by $6 \mathrm{~mm}$ full skin biopsies for measurement of ear weight. Afterwards, mice were euthanized with overdose of isoflurane, and ear tissue samples were taken and fixed with $4 \%$ formaldehyde in phosphate-buffered saline (PBS) and embedded in paraffin. Ear thickness and weight were expressed as percentage of normal controls.

2.1.3. Q-PCR for $m R N A$ Expression. For analysis of the mRNA expression, ear samples were taken after the measurement of ear thickness and weight. Total RNA was isolated from whole ear, using TRI Reagent (Sigma). First strand cDNA was synthesized from $1 \mu \mathrm{g}$ of total RNA with the PrimeScript RT reagent Kit (cDNA Synthesis Kit) (Takara Bio, Japan). The expression levels of mRNA for proinflammatory cytokines were determined by qPCR. The real-time PCR contained $20 \mathrm{ng}$ of reversed transcribed total RNA, $450 \mathrm{nM}$ forward and reverse primers, and $10 \mu \mathrm{l}$ of $2 \mathrm{x}$ LightCycler 480 SYBR Green I Master in a final volume of $20 \mu \mathrm{l}$ in 96-well plates using Bio-Rad CFX96 connect Real-Time PCR System (Bio-Rad, California, USA). Quantification was performed by the comparative $\mathrm{C}_{\mathrm{T}}$ method with mouse GADPH used for normalization. Primer sequences are listed in supplemental Table 1. The relative expression of the mRNAs compared to mRNA in normal mice was calculated. Data are expressed as percentage of normal controls (setting normal controls as $100 \%$ ) [31].

2.1.4. Statistics. Data are expressed as the mean + SEM. The GraphPad Prism 5 software (GraphPad Software, La Jolla, CA, USA) was used for all statistical analyses. One-Way ANOVA with Tukey correction was used to determine the significances when three or more groups were compared. Unpaired two-tailed student's $t$-test with Mann-Whitney test correction was used to determine the statistical significances when two groups.

\section{Results}

We first measured ear thickness and weight, indicators of ear swelling. As shown in Figure 1, topical application of either TPA or DNFB markedly increased both ear thickness (Figure 1(a); $p<0.001$ normal vs. either TPA or DNFB alone) and ear weight (Figure 1(b); $p<0.001$ normal vs. either TPA or DNFB alone). Both topical emollient and hydrocortisone significantly reduced ear thickness and ear weight in both TPA and DNFB models. Notably, the magnitude of reductions in ear thickness was comparable between emollient and 1\% hydrocortisone in both TPA and DNFB models (Figure 1(a)). Similarly, the extent of reduction in ear weight did no differ significantly between emollient and hydrocortisone in TPA model, while ear weight in hydrocortisone-treated group was lower than that in the emollient-treated group in DNFB model (Figure 1(b); $p<$ 0.05). Emollient-induced reductions in ear thickness and ear weight were accompanied with decreased inflammatory infiltration (Figure 2). These results indicate that this novel emollient and hydrocortisone exhibit comparable efficacy in reductions in ear swelling in murine models of acute contact dermatitis.

We next assessed the expression levels of mRNA for proinflammatory cytokines. In allergic contact dermatitis model, 


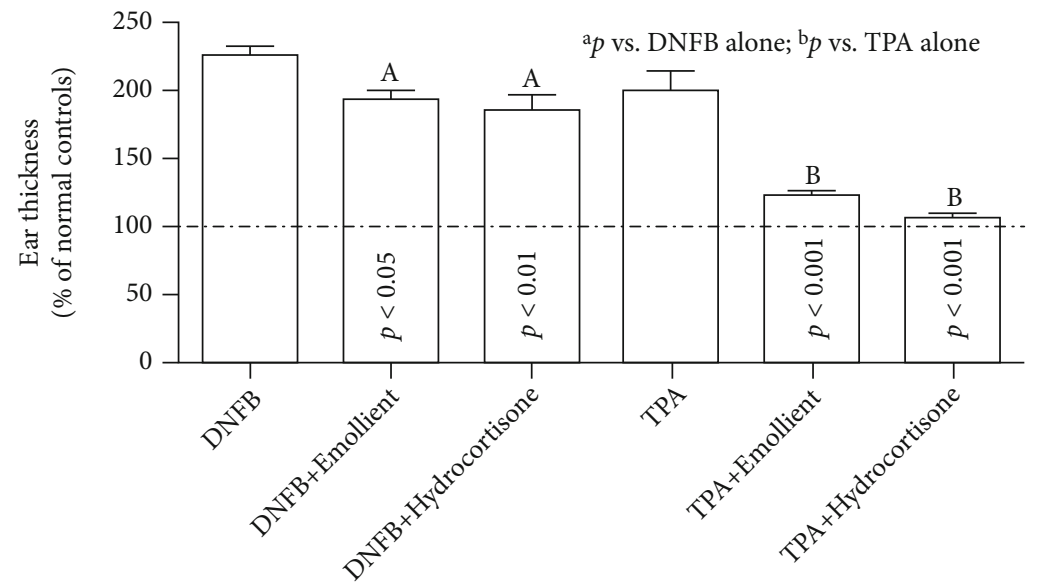

(a)

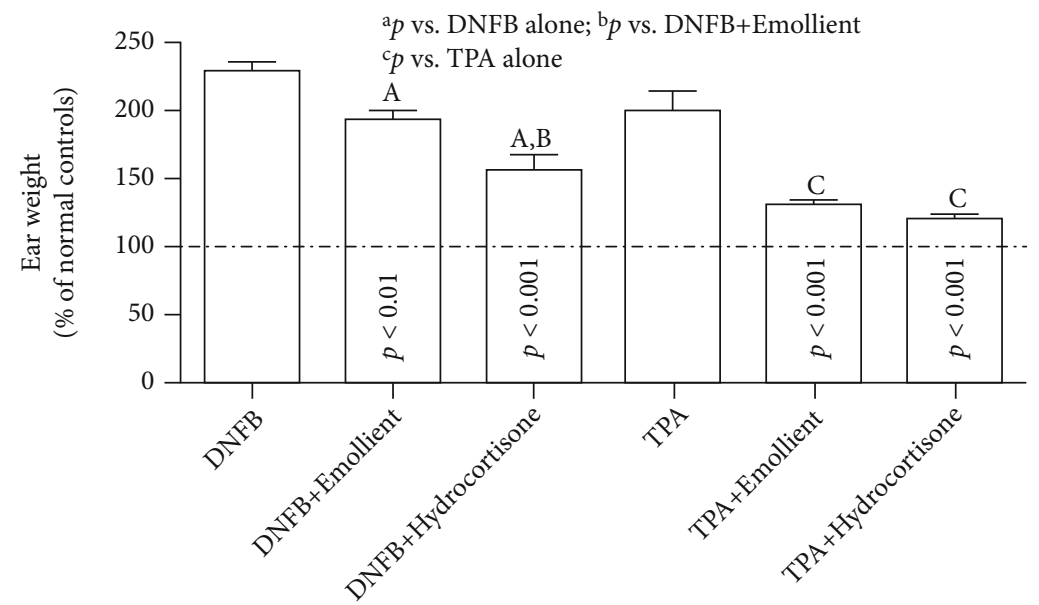

(b)

Figure 1: Topical emollient decreases ear weight and thickness in murine models of acute contact dermatitis: (a, b) depict ear thickness and weight, respectively. Data are expressed as \% of normal controls, setting normal controls as $100 \%$. Significances are indicated in the figures. $N=5$ for all in irritant contact dermatitis model. In allergic contact dermatitis model, $N=6$ for normal controls and $N=7$ for all others.

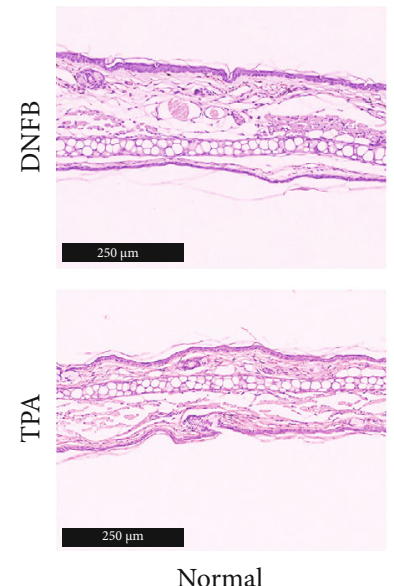

Normal
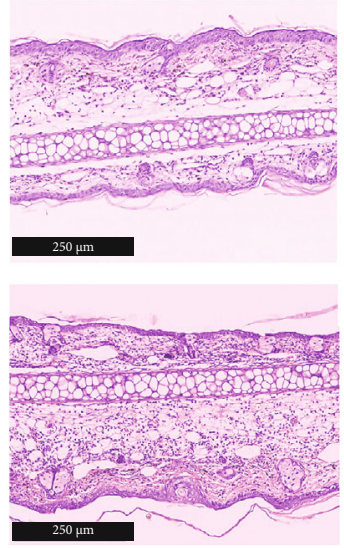

No treatment

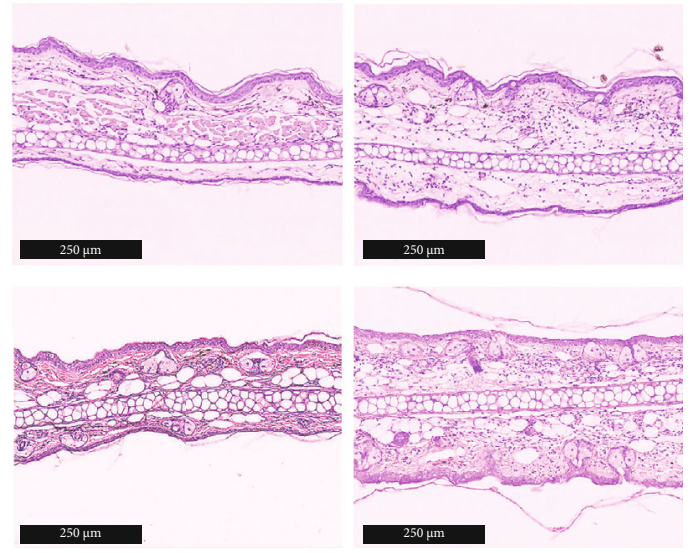

Hydrocortisone
Emollient

Figure 2: Histology of the skin. Skin samples were taken immediately after measurement of ear thickness as described in Materials and Methods. Scale bar $=250 \mu \mathrm{m}$ for all. 


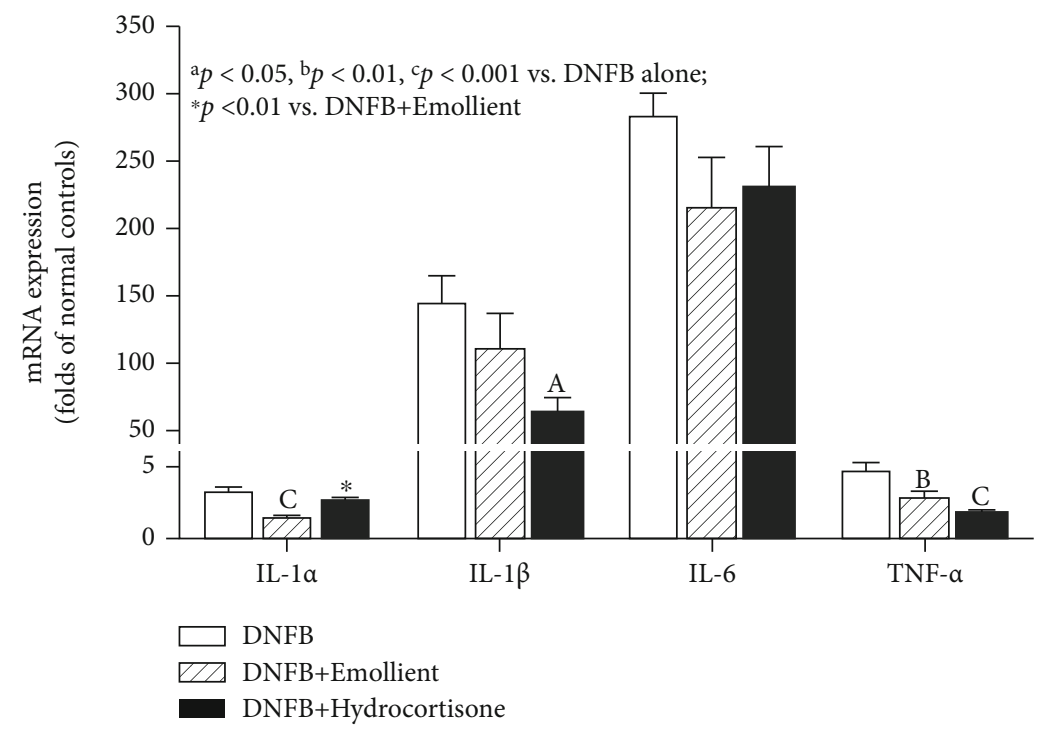

(a) Allergic contact dermatitis

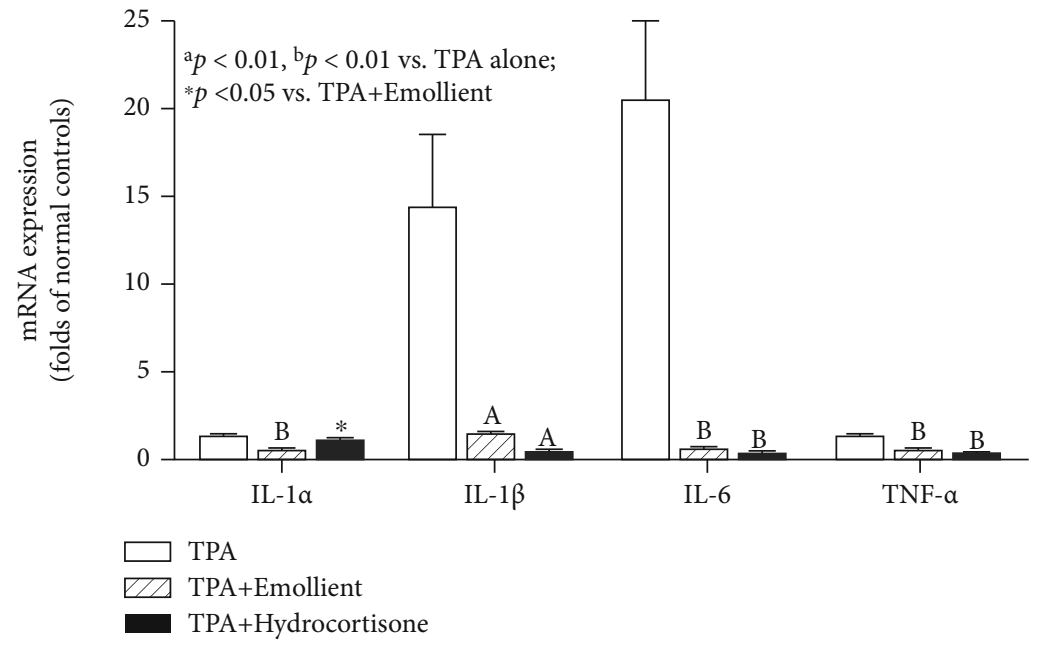

(b) Irritant contact dermatitis

FIGURE 3: Topical emollient decreases expression levels of mRNA for proinflammatory cytokines: (a, b) display expression levels of mRNA for proinflammatory cytokines in allergic and irritant contact dermatitis model, respectively. Data are expressed as folds of normal controls, setting normal controls as 1 . Significances are indicated in the figures. $N=5$ for all in irritant contact dermatitis model. In allergic contact dermatitis model, $N=6$ for normal controls and $N=7$ for all others.

DNFB treatment increased expression levels of mRNA for proinflammatory cytokines (Figure 3(a); DNFB alone vs. normal controls $p<0.001$ for all cytokines). Treatment with hydrocortisone significantly lowered expression levels of IL$1 \alpha$, IL- $1 \beta$, and TNF $\alpha$ but not IL-6. Expression levels of mRNA for IL- $1 \alpha$ and TNF $\alpha$ were also significantly decreased following the treatment with the emollient, without significant changes in expression levels of IL- $1 \beta$ and IL-6. In irritant contact dermatitis model, expression levels of mRNA for IL- $1 \alpha$, IL- $1 \beta$, and IL- 6 were also markedly elevated following TPA treatment, but the levels of TNF $\alpha$ mRNA were not increased significantly (Figure $3(\mathrm{~b})$; TPA alone vs. normal controls, $p<0.05$ to $p<0.001)$. Treatment with emollient lowered expression levels of mRNA for all four cytokines in TPA model. Surprisingly, 1\% hydrocortisone did not reduce the expression levels of IL- $1 \alpha$ mRNA.
Together, these results demonstrated that topical applications of this novel emollient inhibit cutaneous inflammation in mouse models of acute contact dermatitis.

\section{Discussion}

Eczematous dermatitis is a common skin disease. Currently, glucocorticoids and immunosuppressants are the primary modalities in the treatment of this disorder. Although these products are effective, adverse reactions limit their use. Evidence indicates that emollients can be used as adjuvant interventions in the management of eczematous dermatitis $[4,8$, $32,33]$. We showed here that a novel emollient inhibits acute contact dermatitis in mice. It has been presumed that the benefits of emollients for eczematous dermatitis are attributable to the improvements in epidermal permeability barrier 
and stratum corneum hydration [34-36]. This presumption can hold true in chronic dermatitis with defective epidermal permeability barrier and reduced stratum corneum hydration. However, both transepidermal water loss rates and stratum corneum hydration levels are normal in acute dermatitis [37], suggesting that other mechanisms contribute to the inhibition of inflammation by this novel emollient. One potential mechanism can be due to activation of peroxisome proliferator-activated receptors (PPAR) $\alpha$ and $\gamma$ because this novel emollient contains linolenic acid- and linoleic acidenriched natural oils such as Olea europaea oil, Carthamus tinctorius seed oil, and Persea gratissima oil. Previous studies showed that linoleic acid can activate both PPAR $\alpha$ and $\gamma$ [38], while linolenic acid activates PPAR $\gamma$ [39]. Topical applications of either PPAR $\alpha$ or $\gamma$ ligands inhibit cutaneous inflammation in PPAR receptor-dependent manner in acute models of contact dermatitis [28-30]. Thus, inhibition of inflammation by this novel emollient can be ascribable to its activation of PPAR $\alpha$ and $\gamma$.

While glucocorticoids can effectively improve eczematous dermatitis, these products can also compromise epidermal function such as disruption of epidermal permeability barrier function, skin atrophy, and reduction in antimicrobial peptide expression [40-42]. Previous studies demonstrated that topical PPAR ligands prevent glucocorticoidsinduced alterations in epidermal proliferation and permeability barrier function via upregulation of epidermal differentiation and keratinocyte proliferation in normal mouse skin [43]. PPAR $\alpha$ activator alone alleviates cutaneous inflammation in murine model of atopic dermatitis [44]. Combination of glucocorticoids and the $\operatorname{PPAR} \alpha$ activator exhibits superior efficacy to glucocorticoids alone in inhibition of cutaneous inflammation in murine model of atopic dermatitis, while preventing the emergence of glucocorticoidsinduced epidermal side effects [45]. Collectively, a line of evidence suggests a potential utility of emollients containing PPAR ligands in the management of inflammatory dermatoses.

In conclusions, topical application of a novel emollient inhibits cutaneous inflammation in murine models of acute contact dermatitis. Because of the inhibitory effects of this novel emollient on cutaneous inflammation, this novel emollient (possibly other comparable emollients) alone or in combination with glucocorticoids could be a valuable approach in the management of acute and chronic eczematous dermatitis. However, the benefits of this novel emollient for eczematous dermatitis in clinical setting remain to be explored.

\section{Data Availability}

The data that support the findings of this study are available upon reasonable request.

\section{Ethical Approval}

All animal procedures were approved by the Animal Study Subcommittee of the Southern Medical University.

\section{Conflicts of Interest}

The authors have no conflict of interest to declare.

\section{Authors' Contributions}

SW, MS, and LY performed the experiment and approved the final version. BY critically reviewed and interpreted the data and approved the final version. LZH designed the experiment, interpreted the data, and approved the final version. MQM conceived of the presented idea, designed the experiment, analyzed and interpreted the data, drafted the manuscript, and approved the final version.

\section{Acknowledgments}

This work was supported by grants (NSFC 81903188, LY; NSFC 81573075, 81301360, and 81972962, LH) from the National Natural Science Foundation of China and the Medical research foundation of Guangdong province (B2020034, SW).

\section{Supplementary Materials}

Table S1: the primers for QRT-PCR analyses. (Supplementary Materials)

\section{References}

[1] J. I. Silverberg and J. M. Hanifin, "Adult eczema prevalence and associations with asthma and other health and demographic factors: a US population-based study," The Journal of Allergy and Clinical Immunology, vol. 132, no. 5, pp. 11321138, 2013.

[2] D. H. Pols, J. B. Wartna, H. Moed, E. I. van Alphen, A. M. Bohnen, and P. J. Bindels, "Atopic dermatitis, asthma and allergic rhinitis in general practice and the open population: a systematic review," Scandinavian Journal of Primary Health Care, vol. 34, no. 2, pp. 143-150, 2016.

[3] H. O. Skjerven, E. M. Rehbinder, R. Vettukattil et al., "Skin emollient and early complementary feeding to prevent infant atopic dermatitis (PreventADALL): a factorial, multicentre, cluster-randomised trial," Lancet, vol. 395, pp. 951-961, 2020.

[4] J. R. Chalmers, R. H. Haines, L. E. Bradshaw et al., "Daily emollient during infancy for prevention of eczema: the BEEP randomised controlled trial," Lancet, vol. 395, no. 10228, pp. 962-972, 2020.

[5] D. McClanahan, A. Wong, S. Kezic et al., "A randomized controlled trial of an emollient with ceramide and filaggrinassociated amino acids for the primary prevention of atopic dermatitis in high-risk infants," Journal of the European Academy of Dermatology and Venereology, vol. 33, no. 11, pp. 2087 2094, 2019.

[6] T. M. Weber, M. J. Babcock, J. H. Herndon Jr. et al., "Steroidfree emollient formulations reduce symptoms of eczema and improve quality of life," Journal of Drugs in Dermatology, vol. 13, no. 5, pp. 589-595, 2014.

[7] T. M. Weber, J. H. Herndon Jr., M. Ewer et al., "Efficacy and tolerability of steroid-free, over-the-counter treatment formulations in infants and children with atopic dermatitis," Journal 
of the Dermatology Nurses' Association, vol. 7, no. 1, pp. 17-24, 2015.

[8] B. Eberlein, C. Eicke, H. W. Reinhardt, and J. Ring, "Adjuvant treatment of atopic eczema: assessment of an emollient containing N-palmitoylethanolamine (ATOPA study)," Journal of the European Academy of Dermatology and Venereology, vol. 22, no. 1, pp. 73-82, 2008.

[9] I. Angelova-Fischer, G. Neufang, K. Jung, T. W. Fischer, and D. Zillikens, "A randomized, investigator-blinded efficacy assessment study of stand-alone emollient use in mild to moderately severe atopic dermatitis flares," Journal of the European Academy of Dermatology and Venereology, vol. 28, pp. S9-S15, 2014.

[10] E. L. Simpson, J. R. Chalmers, J. M. Hanifin et al., "Emollient enhancement of the skin barrier from birth offers effective atopic dermatitis prevention," The Journal of Allergy and Clinical Immunology, vol. 134, no. 4, pp. 818-823, 2014.

[11] K. Horimukai, K. Morita, M. Narita et al., "Application of moisturizer to neonates prevents development of atopic dermatitis," The Journal of Allergy and Clinical Immunology, vol. 134, no. 4, pp. 824-830.e6, 2014.

[12] T. M. Weber, F. Samarin, M. J. Babcock, A. Filbry, and F. Rippke, "Steroid-free over-the-counter eczema skin care formulations reduce risk of flare, prolong time to flare, and reduce eczema symptoms in pediatric subjects with atopic dermatitis," Journal of Drugs in Dermatology, vol. 14, no. 5, pp. 478-485, 2015.

[13] P. Msika, C. De Belilovsky, N. Piccardi, N. Chebassier, C. Baudouin, and B. Chadoutaud, "New emollient with topical corticosteroid-sparing effect in treatment of childhood atopic dermatitis: SCORAD and quality of life improvement," Pediatric Dermatology, vol. 25, no. 6, pp. 606-612, 2008.

[14] A. Peserico, G. Städtler, M. Sebastian, R. S. Fernandez, K. Vick, and T. Bieber, "Reduction of relapses of atopic dermatitis with methylprednisolone aceponate cream twice weekly in addition to maintenance treatment with emollient: a multicentre, randomized, double-blind, controlled study," The British Journal of Dermatology, vol. 158, no. 4, pp. 801-807, 2008.

[15] W. P. Tan, S. Suresh, H. L. Tey, L. Y. Chiam, and A. T. Goon, "A randomized double-blind controlled trial to compare a triclosan-containing emollient with vehicle for the treatment of atopic dermatitis," Clinical and Experimental Dermatology, vol. 35, no. 4, pp. e109-e112, 2010.

[16] S. Seité, A. Khemis, A. Rougier, and J. P. Ortonne, "Emollient for maintenance therapy after topical corticotherapy in mild psoriasis," Experimental Dermatology, vol. 18, no. 12, pp. 1076-1078, 2009.

[17] N. Cassano, R. Mantegazza, S. Battaglini, D. Apruzzi, F. Loconsole, and G. A. Vena, "Adjuvant role of a new emollient cream in patients with palmar and/or plantar psoriasis: a pilot randomized open-label study," Giornale Italiano di Dermatologia e Venereologia, vol. 145, no. 6, pp. 789-792, 2010.

[18] X. Li, Q. Yang, J. Zheng et al., "Efficacy and safety of a topical moisturizer containing linoleic acid and ceramide for mild-tomoderate psoriasis vulgaris: a multicenter randomized controlled trial," Dermatologic Therapy, vol. 33, no. 6, article e14263, 2020.

[19] M. Q. Man, L. Ye, L. Hu, S. Jeong, P. M. Elias, and C. Lv, "Improvements in epidermal function prevent relapse of psoriasis: a self-controlled study," Clinical and Experimental Dermatology, vol. 44, no. 6, pp. 654-657, 2019.
[20] T. C. Scharschmidt, M. Q. Man, Y. Hatano et al., "Filaggrin deficiency confers a paracellular barrier abnormality that reduces inflammatory thresholds to irritants and haptens," The Journal of Allergy and Clinical Immunology, vol. 124, no. 3, pp. 496-506.e6, 2009.

[21] L. C. Wood, S. M. Jackson, P. M. Elias, C. Grunfeld, and K. R. Feingold, "Cutaneous barrier perturbation stimulates cytokine production in the epidermis of mice," The Journal of Clinical Investigation, vol. 90, no. 2, pp. 482-487, 1992.

[22] T. Nishijima, Y. Tokura, G. Imokawa, N. Seo, F. Furukawa, and M. Takigawa, "Altered permeability and disordered cutaneous immunoregulatory function in mice with acute barrier disruption," The Journal of Investigative Dermatology, vol. 109, no. 2, pp. 175-182, 1997.

[23] E. Proksch and J. Brasch, "Abnormal epidermal barrier in the pathogenesis of contact dermatitis," Clinics in Dermatology, vol. 30, no. 3, pp. 335-344, 2012.

[24] L. Hu, T. M. Mauro, E. Dang et al., "Epidermal dysfunction leads to an age-associated increase in levels of serum inflammatory cytokines," The Journal of Investigative Dermatology, vol. 137, no. 6, pp. 1277-1285, 2017.

[25] Z. Wang, M. Q. Man, T. Li, P. M. Elias, and T. M. Mauro, "Aging-associated alterations in epidermal function and their clinical significance," Aging, vol. 12, no. 6, pp. 5551-5565, 2020.

[26] Y. Hatano, M. Q. Man, Y. Uchida et al., "Maintenance of an acidic stratum corneum prevents emergence of murine atopic dermatitis," The Journal of Investigative Dermatology, vol. 129, no. 7, pp. 1824-1835, 2009.

[27] H. J. Lee, N. Y. Yoon, N. R. Lee, M. Jung, D. H. Kim, and E. H. Choi, "Topical acidic cream prevents the development of atopic dermatitis- and asthma-like lesions in murine model," Experimental Dermatology, vol. 23, no. 10, pp. 736-741, 2014.

[28] M. Y. Sheu, A. J. Fowler, J. Kao et al., "Topical peroxisome proliferator activated receptor- $\alpha$ activators reduce inflammation in irritant and allergic contact dermatitis models," The Journal of Investigative Dermatology, vol. 118, no. 1, pp. 94-101, 2002.

[29] A. J. Fowler, M. Y. Sheu, M. Schmuth et al., "Liver X receptor activators display anti-inflammatory activity in irritant and allergic contact dermatitis models: liver-X-receptor-specific inhibition of inflammation and primary cytokine production," The Journal of Investigative Dermatology, vol. 120, no. 2, pp. 246-255, 2003.

[30] M. Mao-Qiang, A. J. Fowler, M. Schmuth et al., "Peroxisomeproliferator-activated receptor (PPAR) $-\gamma$ activation stimulates keratinocyte differentiation," The Journal of Investigative Dermatology, vol. 123, no. 2, pp. 305-312, 2004.

[31] G. Man, T. M. Mauro, P. L. Kim et al., "Topical hesperidin prevents glucocorticoid-induced abnormalities in epidermal barrier function in murine skin," Experimental Dermatology, vol. 23, no. 9, pp. 645-651, 2014.

[32] Q. Yang, M. Liu, X. Li, and J. Zheng, "The benefit of a ceramide-linoleic acid-containing moisturizer as an adjunctive therapy for a set of xerotic dermatoses," Dermatologic Therapy, vol. 32, article e13017, 2019.

[33] K. L. Hon, N. H. Pong, S. S. Wang, V. W. Lee, N. M. Luk, and T. F. Leung, "Acceptability and efficacy of an emollient containing ceramide-precursor lipids and moisturizing factors for atopic dermatitis in pediatric patients," Drugs in R\&D, vol. 13, no. 1, pp. 37-42, 2013.

[34] S. W. Goh, A. Jamil, N. Safian, N. Md Nor, N. Muhammad, and N. L. Saharudin, "A randomized half-body, double blind, 
controlled trial on the effects of a $\mathrm{pH}$-modified moisturizer vs. standard moisturizer in mild to moderate atopic dermatitis," Anais Brasileiros de Dermatologia, vol. 95, no. 3, pp. 320325, 2020.

[35] R. Bissonnette, C. Maari, N. Provost et al., "A double-blind study of tolerance and efficacy of a new urea-containing moisturizer in patients with atopic dermatitis," Journal of Cosmetic Dermatology, vol. 9, no. 1, pp. 16-21, 2010.

[36] S. L. Chamlin, J. Kao, I. J. Frieden et al., "Ceramide-dominant barrier repair lipids alleviate childhood atopic dermatitis: changes in barrier function provide a sensitive indicator of disease activity," Journal of the American Academy of Dermatology, vol. 47, no. 2, pp. 198-208, 2002.

[37] M. Q. Man, Y. Hatano, S. H. Lee et al., "Characterization of a hapten-induced, murine model with multiple features of atopic dermatitis: structural, immunologic, and biochemical changes following single versus multiple oxazolone challenges," The Journal of Investigative Dermatology, vol. 128, no. 1, pp. 79-86, 2008.

[38] S. A. Kliewer, S. S. Sundseth, S. A. Jones et al., "Fatty acids and eicosanoids regulate gene expression through direct interactions with peroxisome proliferator-activated receptors alpha and gamma," Proceedings of the National Academy of Sciences of the United States of America, vol. 94, no. 9, pp. 4318-4323, 1997.

[39] W. G. Jiang, A. Redfern, R. P. Bryce, and R. E. Mansel, "Peroxisome proliferator activated receptor $-\gamma(\operatorname{PPAR}-\gamma)$ mediates the action of gamma linolenic acid in breast cancer cells," Prostaglandins, Leukotrienes, and Essential Fatty Acids, vol. 62, no. 2, pp. 119-127, 2000.

[40] S. R. Seo, S. G. Lee, H. J. Lee, M. S. Yoon, and D. H. Kim, "Disrupted skin barrier is associated with burning sensation after topical tacrolimus application in atopic dermatitis," Acta Dermato-Venereologica, vol. 97, no. 8, pp. 957-958, 2017.

[41] M. Kim, M. Jung, S. P. Hong et al., “Topical calcineurin inhibitors compromise stratum corneum integrity, epidermal permeability and antimicrobial barrier function," Experimental Dermatology, vol. 19, no. 6, pp. 501-510, 2010.

[42] J. S. Kao, J. W. Fluhr, M. Q. Man et al., "Short-term glucocorticoid treatment compromises both permeability barrier homeostasis and stratum corneum integrity: inhibition of epidermal lipid synthesis accounts for functional abnormalities," The Journal of Investigative Dermatology, vol. 120, no. 3, pp. 456-464, 2003.

[43] M. Demerjian, E. H. Choi, M. Q. Man, S. Chang, P. M. Elias, and K. R. Feingold, "Activators of PPARs and LXR decrease the adverse effects of exogenous glucocorticoids on the epidermis," Experimental Dermatology, vol. 18, no. 7, pp. 643-649, 2009.

[44] Y. Hatano, M. Q. Man, Y. Uchida et al., "Murine atopic dermatitis responds to peroxisome proliferator-activated receptors $\alpha$ and $\beta / \delta$ (but not $\gamma$ ) and liver X receptor activators," The Journal of Allergy and Clinical Immunology, vol. 125, no. 1, pp. 160-169.e5, 2010.

[45] Y. Hatano, P. M. Elias, D. Crumrine, K. R. Feingold, K. Katagiri, and S. Fujiwara, "Efficacy of combined peroxisome proliferator-activated receptor- $\alpha$ ligand and glucocorticoid therapy in a murine model of atopic dermatitis," The Journal of Investigative Dermatology, vol. 131, no. 9, pp. 1845-1852, 2011. 